\title{
Análise comparativa da percepção da população sobre o diagnóstico clínico e o diagnóstico psicológico.
}

\section{Comparative analysis of the population's perception of clinical and psychological diagnoses.}

Fernanda Gonçalves da Silva ${ }^{1 *}$, Pâmela Cristine dos Santos Bastos da Fonseca² ${ }^{2}$ Cíntia Cassimiro da Silva ${ }^{2}$, Ana Carolina Carmo Fernandes ${ }^{2}$, Beatriz da Cunha Corrêa ${ }^{2}$, Marcos Vinicius Oliveira da Silva², Vitória do Nascimento Santos², Carla Cristina Braga Santos ${ }^{2}$

\section{RESUMO}

O Psicodiagnóstico é uma avaliação psicológica realizada com objetivos clínicos, a fim de identificar a presença ou não de critérios diagnósticos de psicopatologias nos indivíduos. A avaliação psicológica clínica possui um papel fundamental na prevenção e promoção de saúde mental. O presente artigo analisou a correlação do temor da população a respeito do psicodiagnóstico comparado com o temor do diagnóstico de patologias médicas. Foi realizada uma pesquisa transversal e exploratória, onde através de um formulário online, os dados foram coletados por mídias sociais no período de dezembro de 2020 a maio de 2021. Os resultados apontaram para um maior temor da população a respeito do diagnóstico médico, visto que $58,8 \%$ consideraram o diagnóstico médico mais grave, e 41,2\% julgam o diagnóstico psicológico como sendo o mais grave. Concluindo pode-se analisar que o sofrimento psíquico ainda é constantemente encarado como algo menos relevante, tratado por vezes à base somente de medicação em razão do saber presente no discurso médico e no imediatismo, o que interfere diretamente nessa percepção de maior temor. É proposto que maiores pesquisas analisem outras variáveis que interferem nessa percepção para inferências que auxiliem nesse contexto.

Palavras-chave: Psicodiagnóstico; Psicopatologia; Avaliação Psicológica; Diagnóstico Médico; Análise comparativa.

\begin{abstract}
Psychodiagnosis is a psychological assessment carried out with clinical objectives, to identify the presence or absence of diagnostic criteria for psychopathologies in individuals. Clinical psychological assessment plays a key role in the prevention and promotion of mental health. The present article analyzed the correlation of the population's fear regarding psychodiagnosis compared with the fear of the diagnosis of medical pathologies. A cross-sectional and exploratory research was carried out, where, through an online form, data were collected by social media from December 2020 to May 2021. The results pointed to a greater fear of the population about the medical diagnosis, since $58.8 \%$ considered the medical diagnosis to be the most serious, and $41.2 \%$ considered the psychological diagnosis to be the most serious. In conclusion, it can be analyzed that psychic suffering is still constantly seen as something less relevant, sometimes treated only with medication due to the knowledge present in the medical discourse and
\end{abstract}

\footnotetext{
${ }^{1}$ Universidade Federal do Rio de Janeiro 1. *E-mail: fernandagoncalves.fgs@gmail.com

2 Universidade Estácio de Sá
} 
immediacy, which directly interferes with this perception of greater fear. It is proposed that further studies analyze other variables that interfere in this perception for inferences that help in this context.

Keywords: Psychodiagnosis; Psychopathology; Psychological Assessment; Medical diagnostic; Comparative analysis.

\section{INTRODUÇÃO}

A Organização Mundial de Saúde (OMS, 1946) reconhece o bem-estar mental como um fator indispensável para o indivíduo ter saúde. Em 2001, no relatório mundial da OMS, a saúde mental outrora negligenciada e estigmatizada, se torna equivalente aos demais aspectos, definindo-a de modo geral como bem-estar subjetivo do funcionamento das áreas cognitivas, emocionais, comportamentais, de autonomia, da percepção de sua autoeficácia e outros domínios (Relatório Mundial de Saúde, 2001).

O Mental Health Atlas apresenta planos de ação e perspectivas a fim de restringir mundialmente os impactos de saúde mental, buscando um fortalecimento de valores, princípios, objetivos e de áreas, para uma promoção e prevenção de saúde mental. O total de países que responderam aos relatórios, mesmo que parcialmente, correspondem a porcentagem de 97,5\% da população mundial, ganhando notoriedade em suas ações. Em 2015, a saúde mental passa a compor um dos objetivos na Agenda de Desenvolvimento Sustentável, tendo como perspectiva diminuir um terço da mortalidade prematura de doenças não transmissíveis e promoção de saúde mental e bem-estar até 2030. Dentre as metas para alcançar essa perspectiva está a construção de programas de prevenção que possam contribuir para a redução de estigmas, discriminação e violação dos direitos humanos (Mental Health Atlas, 2017; Organização Mundial da Saúde; 2018).

O Manual Diagnóstico e Estatístico de Transtornos Mentais (DSM-V) desde 1844, se empenha em estudos científicos e estatísticos em uma equipe multidisciplinar de saúde, com intuito de auxiliar em planos de tratamentos mais consistentes para cada indivíduo. Essas diferentes áreas da saúde, buscam compreender as psicopatologias que impactam a saúde mental da população mundial de diversas concepções, abarcando conhecimentos sobre prevalências, sintomas, fatores de risco, transmissões familiares e influências sociais nos desdobramentos (American Psychological Association) [APA], 2014).

A psicologia é atuante nesse movimento científico e o psicodiagnóstico é a área que desempenha papel relevante frente a esses diagnósticos psicológicos (Tavares, 2004). 
O psicodiagnóstico é realizado por psicólogos em contexto clínico, no qual dedicam-se na compreensão do sujeito e todas as suas perspectivas, a fim de mensurar diversos constructos que identifiquem a presença ou não de psicopatologia. Esse processo de mensuração partirá de hipóteses pressupostas pelo psicólogo, realizando-a através da integração dos dados obtidos por técnicas, testes, entrevistas e outros instrumentos psicológicos que possam ser necessários na formulação do plano de avaliação de cada avaliado, refutando ou confirmando as hipóteses iniciais (Cunha, 2003).

Em 2004, Tavares apresenta a avaliação psicológica como um processo de sapiência e não rotulagem de patologias, reconhecendo que esse objetivo advém do intuito de promover saúde aos indivíduos diagnosticados, para evolução de quadros clínicos e autoconhecimento.

Essa compreensão holística do sujeito, ou seja, entender integralmente seus fenômenos existentes, permitirá no psicodiagnóstico atingir vários objetivos. Destaca-se dentre eles, a identificação precoce de possíveis fatores predisponentes, que por experiências interpessoais ou percepções individuais, podem desencadear transtornos psicológicos, servindo de prevenção de possíveis psicopatologias. Portanto a avaliação psicológica clínica possui diferentes benefícios, em casos de uma classificação nosológica, onde foi identificado um transtorno mental, o prognóstico servirá como um potencial redutor de desdobramentos negativos, visto que reconhecer as probabilidades de desencadeamentos do caso, permitirá desenvolver estratégias para extinguir ou reduzir os sintomas (Cunha, 2003).

Frequentemente, pacientes adolescentes encaminhados para o Psicodiagnóstico apresentam dificuldades relacionadas à aprendizagem, afetos e agressividade (Cunha \& Benetti 2009; Santos 2006). Ao que se refere à avaliação dos adultos as maiores queixas são problemas de relacionamento familiar (Louzada, 2003; Maravioski \& Serralta, 2011). Outros profissionais podem subsidiar a conclusão de uma hipótese diagnóstica, tais como: Fonoaudiólogos, Neurologistas, Psiquiatras, Psicopedagogos, que irão auxiliar na realização de exames complementares, isso se configura como uma responsabilidade compartilhada, no entanto, o fechamento do diagnóstico psicológico será sempre responsabilidade exclusiva do psicólogo. Este processo implica identificar e medir as potências e fragilidades do funcionamento psíquico, considerando os critérios de normalidade (Cunha, 2003). 
Visto a relevância da avaliação psicológica clínica e suas contribuições na prevenção e promoção de saúde e a lacuna existente de dados que apresentem a maneira que a população percebe o fenômeno. $\mathrm{O}$ objetivo desse estudo se propôs a investigar a percepção da população frente aos transtornos psicológicos e analisar o temor mediante a esses, comparados com patologias médicas, no qual as informações obtidas nortearão propostas mais eficientes, a fim de reduzir os possíveis temores diante aos diagnósticos.

\section{METODOLOGIA}

\section{Participantes}

A amostra foi composta por 169 participantes, dos quais 10 por ausências e incongruências nas respostas, foram excluídos. Permaneceu assim, 159 participantes para análise de dados, que responderam corretamente às perguntas dos formulários. A amostra apresentou maior representatividade do gênero feminino com 76,4\%, região Sudeste com 94,3\% e que tinham ensino superior incompleto $38 \%$.

\section{Procedimentos}

Esta é uma pesquisa transversal e exploratória, no qual foi desenvolvido um formulário online pelos pesquisadores do Grupo de Pesquisa em Avaliação Psicológica (GPAP), onde os dados foram coletados por mídias sociais no período de dezembro de 2020 a maio de 2021. O formulário disponibilizado continha perguntas para coleta de informação sociodemográfica, como gênero, escolaridade, profissão, estado e religião, além da captação das respostas referentes ao temor do diagnóstico psicológico ou médico, onde eram respondidas após aceitarem o Termo de Consentimento Livre e Esclarecido TCLE.

Ao que se refere às perguntas com escolha forçada sobre o diagnóstico mais temido, os participantes responderam a 20 questões contendo duas alternativas cada, sendo 19 dessas comparando duas patologias específicas, e uma indagando qual o tipo de diagnóstico é mais grave, psicológico ou médico.

Análise de dados

Os dados foram coletados pelo formulário e transportados para uma planilha no Microsoft Excel, na qual foram analisadas em percentuais as perguntas sociodemográficas dividindo-as em gênero, escolaridade, região e religião, que pode ser observado na tabela 1 . 
Foram analisados os dados coletados sobre a comparação das respostas forçadas às perguntas sobre diagnósticos psicológicos e médicos. Realizou-se também as correlações entre gênero, escolaridade e os temores diagnósticos, nos quais ambos foram feitos gráficos para melhor compreensão dos resultados.

\section{RESULTADOS}

Nos resultados, pode-se observar na tabela 1 os quantitativos respondentes de cada item e os percentuais sobre os dados sociodemográficos, apresentando abaixo informações a respeito do gênero, escolaridade, região e religião da amostra.

Tabela 1- Resultados com dados a respeito de gênero, escolaridade, região e religião.

\begin{tabular}{llll}
\hline & F & \% \\
& & & \\
\hline \multirow{2}{*}{ Escolaridade } & Ensino Superior Incompleto & 61 & $38 \%$ \\
& Ensino Superior Completo & 31 & $19 \%$ \\
& Pós-graduado & 29 & $18 \%$ \\
& Ensino Médio Completo & 25 & $16 \%$ \\
& Mestre & 8 & $5 \%$ \\
& Ensino Médio Incompleto & 4 & $3 \%$ \\
Fundamental Completo & 1 & $1 \%$ \\
& & \\
Região & 150 & $94,3 \%$ \\
& Sudeste & 5 & $3,1 \%$ \\
& Nordeste & 2 & $1,3 \%$ \\
Norte & 1 & $0,6 \%$ \\
Centro-oeste & 1 & $0,6 \%$ \\
Sul & & \\
& & \\
\hline
\end{tabular}

Os dados apontam que 58,8\% dos participantes consideram o diagnóstico médico mais grave, enquanto 41,2\% da amostra julgam ser o diagnóstico psicológico. Esse dado vai de encontro à uma de nossas hipóteses iniciais, mostrando que os participantes de fato 
percebem o diagnóstico médico como mais perigoso. Contando as 19 perguntas sobre patologias específicas, o diagnóstico médico é mais temido em 13 delas, sendo o diagnóstico psicológico mais temido em apenas 6, como demonstra a Figura 1.

Figura $1^{2}$ - Comparação entre diagnósticos médicos e psicológico

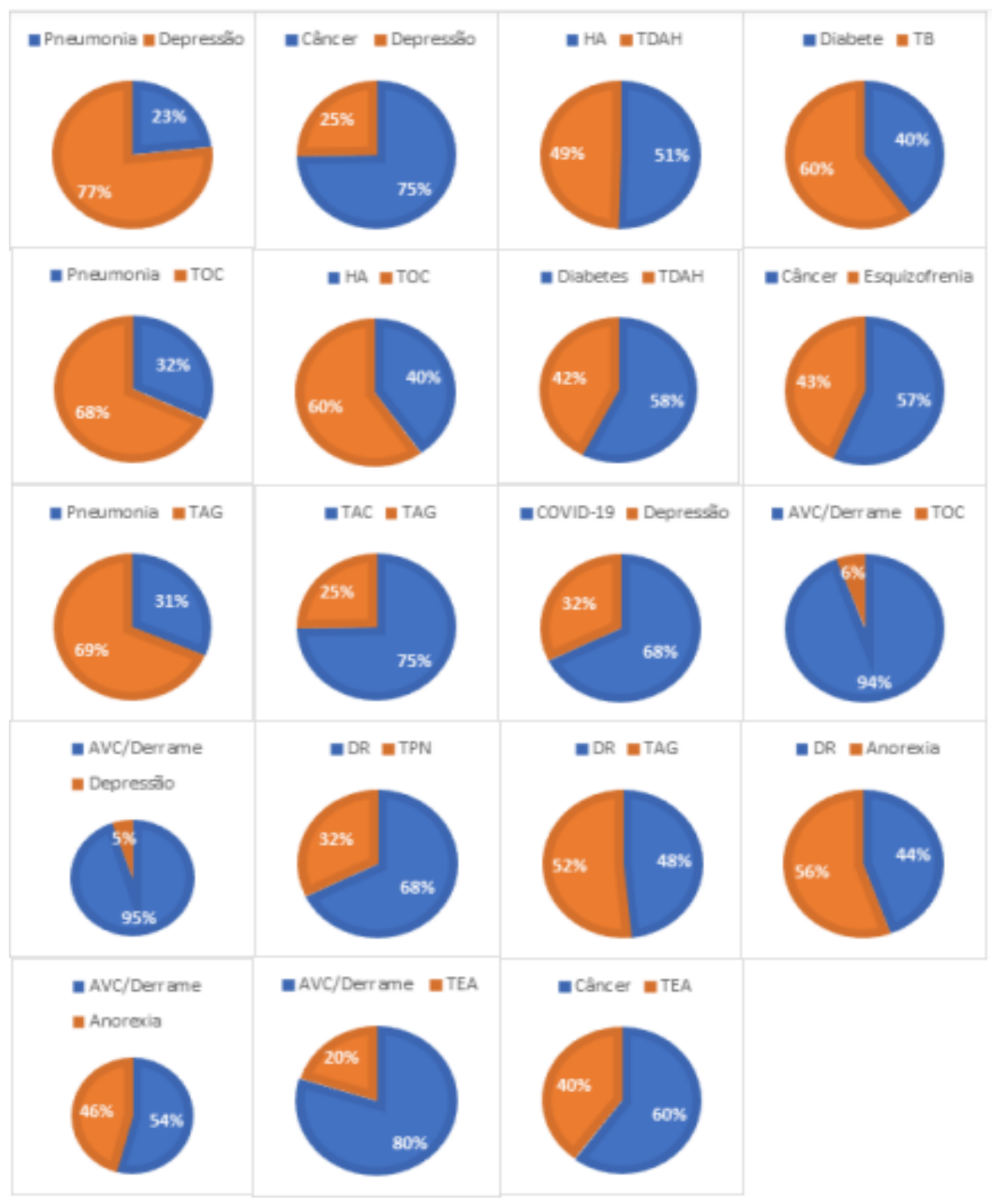

Figura 2 - Correlação entre a escolaridade e o medo do diagnóstico médico e psicológico.

2 Transtorno Bipolar (TB), Hipertensão Arterial (HA) e Transtorno do Déficit de Atenção com Hiperatividade (TDAH), Transtorno Obsessivo Compulsivo (TOC), Transtorno de Ansiedade Generalizada (TAG), Doença Arterial Coronariana (DAC), Acidente Vascular Cerebral (AVC)/Derrame, Doença Renal (DR) e Transtorno de Personalidade Narcisista (TPN), Acidente Vascular Cerebral (AVC)/Derrame e Transtorno do Espectro Autista (TEA). 


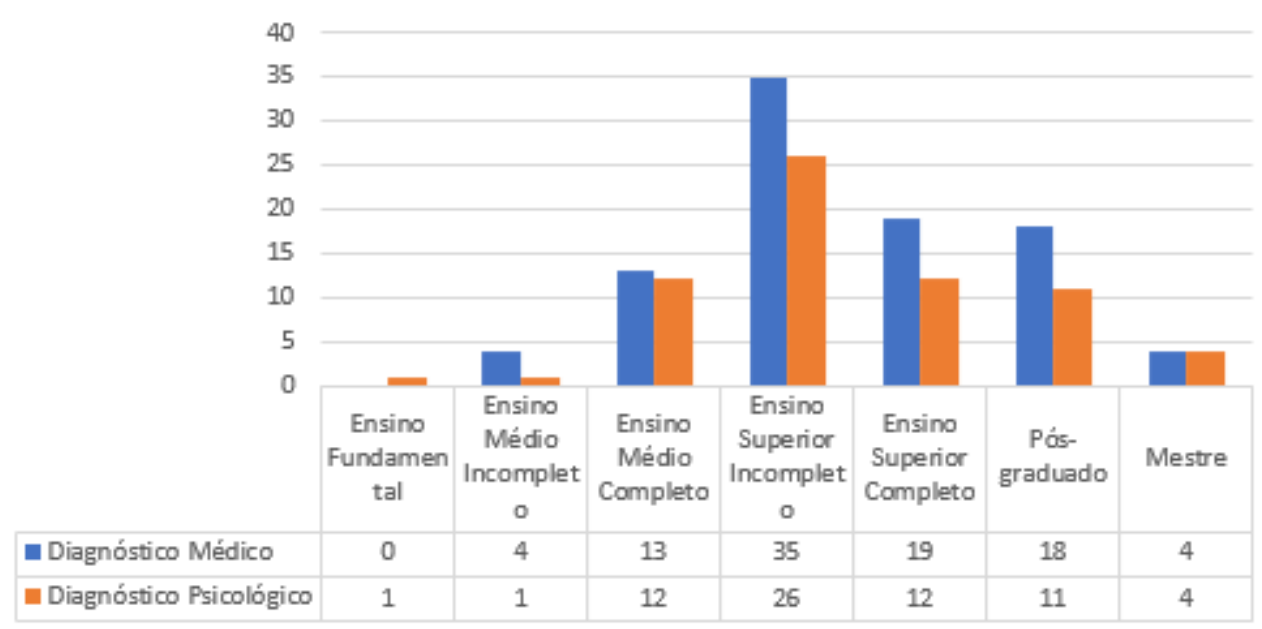

Na Figura 2, a população com escolaridade Ensino Médio Incompleto, Ensino Superior Incompleto, Ensino Superior Completo e Pós-graduado dizem temer mais o diagnóstico médico que o diagnóstico psicológico, sendo Ensino Superior Incompleto, Ensino Superior Completo e Pós-graduado, os que tiveram maior destaque comparado aos outros, como Ensino Médio Incompleto e Ensino Médio Completo.

Figura 3 - Correlação entre gênero e temor do psicodiagnóstico.

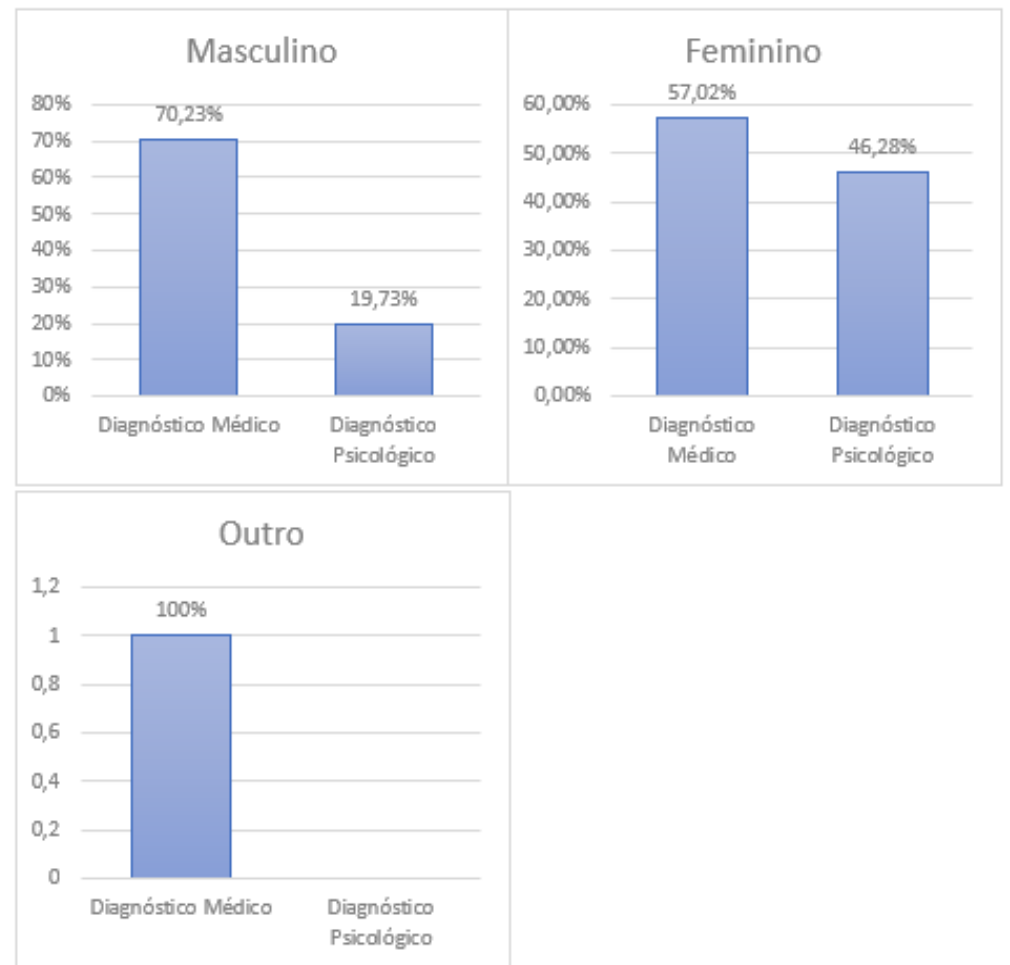

Observamos que 57,02\% do gênero Feminino mostrou maior temor pelo diagnóstico médico, enquanto 46,28\% apresentaram maior temor para diagnóstico psicológico. Analisando somente o gênero masculino apontou para 70,23\% dos 
participantes apresentando maior temor para diagnóstico médico, quando apenas 19,73\% apresentaram maior temor para o diagnóstico psicológico. Em "Outros", a porcentagem de maior temor para diagnóstico médico é de $100 \%$.

\section{DISCUSSÃO}

Os resultados desta pesquisa elucidaram informações sobre as percepções dos indivíduos mediante ao psicodiagnóstico comparados com o diagnóstico médico. Ainda que as contribuições do psicodiagnóstico sejam relevantes para promoção de saúde, ora para identificação de transtornos psicológicos ou para fatores que possam desencadeálos, na percepção da população, as psicopatologias apresentou-se sendo o mais temido em apenas $41,2 \%$ da amostra representativa.

Dentre os dados extraídos da amostra, no que se refere ao gênero e a escolha entre os tipos de diagnósticos, percebeu-se que os homens temem mais ao diagnóstico médico, apontando uma discrepância de cerca de 50\% em comparação ao diagnóstico psicológico, enquanto diferença entre as mulheres é aproximadamente $10 \%$.

Embora o gênero masculino em uma pesquisa realizada por Alves et al., (2011) tenha sido o público com baixa procura dos serviços de saúde, sendo perpassados por concepções culturais de um grupo que é invulnerável e forte, são os mesmo com maior temor em diagnósticos médicos, quando comparados com o gênero feminino que é considerado como sendo mais cuidadoso. (Alves et al., 2011). Podendo inferir que essas percepções possam ser permeadas por construções culturais, nas quais transtornos psicológicos acometem pessoas mais vulneráveis.

O Relatório Mundial da Saúde (2002) sugere que o estigma em torno da saúde mental é resultado do medo pertinente à falta de informação sobre os transtornos mentais e possui grande impacto negativo para o sujeito adoecido, fazendo com que o mesmo não busque o tratamento necessário. Informações sobre saúde mental precisam ser acessíveis para a população, com o objetivo de diminuir o medo relacionado aos transtornos e consequentemente uma melhora na qualidade de vida.

O estigma também pode ser considerado uma barreira para a aceitabilidade de que transtornos mentais, levando pessoas a serem excluídas do convívio social por suas psicopatologias. Além disso, o estigma reduz o acesso ao tratamento adequado, o que 
enfatiza o desespero e os processos autodestrutivos (Department of Health and Human Services) [DHHS], 1999).

Através da correlação entre escolaridade e temor diagnóstico, foi possível identificar que 79,4\% dos participantes possuíam escolaridade a partir do ensino superior incompleto e nas correlações do ensino médio incompleto ao pós-graduado o diagnóstico médico foi o mais temido. Presumindo-se que possa existir um desconhecimento dos graduandos, graduados e pós-graduados ao que se refere a saúde mental e as implicações dos transtornos e ao diagnóstico psicológico, temendo apenas as doenças.

Observou-se que os transtornos psicológicos quando comparados a diagnósticos como: Depressão com Pneumonia, TB com Diabetes, TDAH com Hipertensão, TOC com Pneumonia, TOC com Hipertensão e TAG com Pneumonia, apresentam maior temor, mesmo que com uma diferença percentual pequena em alguns.

O TOC pode gerar uma ansiedade aflitiva no indivíduo, em que se comportam com repetidas atitudes, a fim de reduzir a ansiedade gerada, agindo compulsivamente (Vivian, 2013; APA, 2014). Ao contrapor com os diagnósticos de Pneumonia e Hipertensão que são doenças que obtém tratamentos acessíveis, o diagnóstico de TOC foi mais temido por cerca de $60 \%$ dos participantes, mas quando equiparado ao AVC somente $5,5 \%$ dos participantes dizem temê-lo.

O Transtorno de Ansiedade possui os mais variados sintomas, que por vezes podem ser confundidos com patologias médicas, a exemplo da taquicardia (CID-10). A Depressão por sua vez, apresenta desde humor deprimido a redução da capacidade de cuidar de si e de suas necessidades básicas e dos outros. (APA, 2014; OPAS, 2019; Ritchie \& Roser, 2018). Tais transtornos são bastante explorados na mídia, pode ser que por esse motivo que apareçam na pesquisa com percentual superior a $64 \%$, indicando maior temor quando comparados a doenças como a pneumonia.

Para a comparação Esquizofrenia e Câncer, obteve-se 57\% da amostra dizendo temer mais o diagnóstico médico (câncer), ainda que esse transtorno alcance o rompimento com a realidade. A Esquizofrenia aponta características comuns como delírios, alucinações, sintomas negativos, comportamento motor e pensamentos desorganizados. (APA, 2014)

A pesquisa aponta que $65,5 \%$ dos participantes dizem temer mais o diagnóstico de doença renal que o TPN (34,5\%), mesmo que este impacte em diversas áreas de 
relacionamentos interpessoais e profissionais, por ser um transtorno de personalidade e esteja em comorbidade com outros transtornos. (APA, 2014)

O TDAH promove prejuízos significativos na aprendizagem, desde os primeiros anos escolares à graduação, impactando a autoestima, autoconceito e até mesmo na área profissional (OPAS, 2019; APA, 2014). Tal transtorno foi comparado com três diagnósticos médicos diferentes, hipertensão (49,7\%), diabetes $(58,2 \%)$ e AVC $(94,5 \%)$, mostrando que o diagnóstico médico é mais temido em duas das três comparações.

O TEA trata-se de uma condição de neurodesenvolvimento vitalícia que interfere na capacidade da pessoa de se comunicar e se relacionar com outras pessoas. (APA, 2014). A amostra também evidenciou maior temor dos participantes para doenças como o $\operatorname{AVC}(79,4 \%)$ e câncer $(83,6 \%)$.

Os Transtornos Psicológicos assim discorridos e caracterizados como, o TB, TPN e a Esquizofrenia, podem interferir nos seus relacionamentos familiares, assim como em ambientes que exijam maior habilidade de relações interpessoais, produtividade e concentração (OPAS, 2019; APA, 2014).

O TDAH e TEA impactam diretamente no funcionamento, no qual adquirem conhecimentos acadêmicos, necessitando de adaptações nos modelos de ensino tradicional, para uma aprendizagem eficiente. (APA, 2014)

TAG, TOC e Depressão influenciam diretamente na percepção de si, apresentando-se sintomas incapacitantes momentaneamente contraproducentes, impactando áreas profissionais (OPAS, 2019; APA, 2014).

Assim, como descrito, alguns desdobramentos dos Transtornos investigados, pela ausência de um diagnóstico psicológico ou pelo desconhecimento de suas consequências, podem produzir danos, por vezes até maiores que os sintomas patológicos, ao interferir em áreas de sustento, convívio social e desenvolvimentos psicológicos, pontos pertinentes para um modelo de saúde biopsicossocial.

A população apresenta frequentemente uma visão reducionista sobre transtornos mentais. Isso deve-se a história, na qual construiu-se uma percepção popular de que os transtornos mentais eram sobrenaturais e perigosos. Essa visão reducionista foi construída limitadamente não só na sociedade a respeito dos transtornos mentais ou das pessoas próximas, mas também dos próprios profissionais de saúde, que se preocupavam apenas em medicar ao invés de investigar, ouvir e acolher a queixa do paciente. (Costa \& Finelli, 2016). 
No entanto, referindo-se a medicina ainda existem discussões acerca da dificuldade em lidar com o que é subjetivo e que foge ao físico e localizável (Farias et al., 2021). O sofrimento psíquico frequentemente é encarado como algo menos relevante, tratado por vezes à base somente de medicação em razão do saber presente no discurso médico e no imediatismo (Cavalcanti, 2005). O poder do discurso biomédico tem sua força potencializada pela internalização feita por pacientes que reteve esse saber por séculos como único e verdadeiro (Farias et al., 2021).

Infere-se que a sociedade que não entende transtorno mental, tampouco entende o psicodiagnóstico. A partir daí pode-se compreender a construção da hipótese de que a percepção social enxerga o diagnóstico como algo mais perigoso que o psicodiagnóstico, porque observa-se que o transtorno médico é frequentemente visto como algo biológico e "não-evitável", e os transtornos psicológicos aparecem em pessoas mais "fracas" ou que possuem uma predisposição à "loucura" ou ao "sobrenatural" pela construção histórica.

É possível apontar para algumas limitações neste estudo, no qual não foi analisado os níveis de complexidade de cada patologia, sendo o critério de escolhas forçadas, onde a amostra teve que escolher entre um ou outro, portanto essas opções de escolhas foram feitas de forma randômica, não identificando possíveis variáveis que possam influenciar essas escolhas. Para a redução desse viés, as psicopatologias foram repetidas e comparadas com outras doenças, a fim de identificar a real percepção da população.

Sendo assim, é necessário que mais pesquisas sejam elaboradas com intuito de verificar as variáveis que possam impactar nessas escolhas, assim como os níveis de complexidade e acesso a tratamentos dessas doenças, identificando fatores de intervenção nesse processo de temor e medo, como também o desconhecimento dos desdobramentos dos transtornos psicológicos e acesso ao psicodiagnóstico.

\section{REFERÊNCIAS}

ALLAN, Chris M. et al. A conceptual model for interprofessional education: The International Classification of Functioning, Disability and Health (ICF). Journal of Interprofessional Care, v. 20, n. 3, p. 235-245, 2006

ALVES, Railda Fernandes et al. Gênero e saúde: o cuidar do homem em debate. Psicologia: teoria e prática, São Paulo, v. 13, n. 3, p. 52-1166, 2011.

AMERICAN PSYCHIATRIC ASSOCIATION - APA. Manual diagnóstico e estatístico de transtornos mentais: DSM-5. Porto Alegre: Artmed, 2014. 
CAVALCANTI, Ignez Leturzia. O sujeito da psicanálise em instituições psiquiátricas públicas. Psicanálise, clínica e instituição, p. 169-183, 2005.

CEDRO FARIAS, Isabela et al. " Médico Disse que Era Só Psicológico": Analisando o Lugar da Psicologia no Campo da. Estudos e Pesquisas em Psicologia, v. 21, n. 3, 2021.

CONSELHO FEDERAL DE PSICOLOGIA - CFP. Resolução CFP n⿳0 007/2003. Brasília: CFP, 2003.

COSTA, T. O.; FINELLI, L. A. C. . Percepção Sociohistórica acerca dos Portadores de Sofrimento Mental: Uma Revisão da Literatura. Revista Bionorte, v. 4, p. 11-24, 2016.

CUNHA, Jurema Alcides. Psicodiagnóstico-V. 5 a ed. Porto Alegre: Artmed, 2003.

CUNHA, Tatiane Regina dos Santos; BENETTI, Silvia Pereira da Cruz. Caracterização da clientela infantil numa clínica-escola de psicologia. Boletim de psicologia, São Paulo, v. 59, n. 130, p. 117-127, jun. 2009.

LOUZADA, Rita de Cássia. Caracterização da clientela atendida no Núcleo de Psicologia Aplicada da Universidade Federal do Espírito Santo. Estudos de Psicologia, Natal, v. 8, n. 3, p 451-457, 2003.

MARAVIESKI, Silvinha; SERRALTA, Fernanda Barcellos. Características clínicas e sociodemográficas da clientela atendida em uma clínica-escola de psicologia. Temas em psicologia, Ribeirão Preto, v. 19, n. 2, p. 481-490, dez. 2011.

ORGANIZAÇÃO MUNDIAL DA SAÚDE - OMS. Relatório Mundial da Saúde: Saúde Mental: nova concepção, nova esperança. Genebra: 2002.

ORGANIZAÇÃO PAN-AMERICANA DA SAÚDE. Transtornos Mentais. Brasília, 2019.

RITCHIE, Hannah; ROSER, Max. Mental health. Our world in data. Retrieved May, v. 19, p. 2020, 2018.

TAVARES, Marcelo. Validade Clínica. Psico-USF, v. 8, n. 2, p. 125-136, 2003.

UNITED STATES. PUBLIC HEALTH SERVICE. OFFICE OF THE SURGEON GENERAL et al. Mental health: A report of the surgeon general. Department of Health and Human Services, US Public Health Service, 1999.

VIVIAN, ANALISE DE SOUZA. Prevalência Do Transtorno Obsessivo-compulsivo E De Sintomas Obsessivo-compulsivos E Qualidade De Vida Em Adolescentes. 2008. 71 f. Tese (Mestrado em Ciências Médicas: Psiquiatria) - Universidade Federal do Rio Grande do Sul, Rio Grande do Sul, 2008.

WELLS, R. H. C.; BAY-NIELSEN, H.; BRAUN, R.; et al. CID-10: classificação estatística internacional de doenças e problemas relacionados à saude. [S.l: s.n.], 2011. 
WORLD HEALTH ORGANIZATION - WHO. Mental Health Atlas 2017. Geneva: World Health Organization, 2018.

WORLD HEALTH ORGANIZATION et al. Preamble to the Constitution of the World Health Organization as adopted by the International Health Conference, New York, 1922 June 1946; signed on 22 July 1946 by the representatives of 61 States (Official Records of the World Health Organization, no. 2, p. 100) and entered into force on 7 April 1948.

Recebido em: 05/01/2022

Aprovado em: 08/02/2022

Publicado em: 11/02/2022 